\title{
Mortalidade em receptores de transplante renal - causas e fatores relacionados: revisão integrativa
}

\section{Mortality in renal transplant receptors - causes and related factors: integrative review}

\author{
Willian Alburquerque de Almeida' $\bullet$ Adriano Menis Ferreira ${ }^{2} \bullet$ Marcelo Alessandro Rigotti ${ }^{3}$ \\ Ana Lúcia Queiroz Bezerra ${ }^{4} \bullet$ Adaiele Lúcia Nogueira Viera da Silva ${ }^{5}$ Cristiane Chagas Teixeira ${ }^{6}$
}

\begin{abstract}
RESUMO
Objetivos:Analisar as produções científicas sobre os fatores associados à mortalidade em pacientes submetidos ao transplante renal. Métodos: revisão integrativa realizada no período de janeiro de 2006 a julho de 2016, cujo levantamento bibliográfico foi por meio das bibliotecas eletrônicas: PubMed, SciELO, LILACS e Cochrane Library, totalizando 777 artigos. Após a leitura dos títulos e resumos, foi excluída toda publicação duplicada e as que não correspondiam aos critérios de inclusão.A amostra compôs-se de 21 estudos os quais responderam à questão de pesquisa. Resultados:A maior incidência de publicações deu-se em periódicos internacionais, predominando estudos de coorte com nível de evidência IV. Quanto às causas da mortalidade prevaleceu a cardiovascular. Os fatores associados a mortalidade foram relacionados a função cardiovascular, processos infecciosos, metabólicos, fisiológicos, nutricionais e estruturais. Conclusões: Recomenda-se melhorias no processo de avaliação e acompanhamento dos pacientes receptores de transplante renal.
\end{abstract}

Descritores: Transplante de rim; Mortalidade; Fatores de risco; Complicações pós-operatórias; Revisão.

\begin{abstract}
Objectives:To analyze the scientific production on the factors associated with mortality in patients submitted to renal transplantation. Methods:An integrative review was carried out from January 2006 to July 2016, whose bibliographic survey was done through electronic libraries: PubMed, SciELO, LILACS and Cochrane Library, totaling 777 articles. After reading the titles and abstracts, all duplicate publications and those that did not meet the inclusion criteria were excluded.The sample consisted of 21 studies which answered the question of research. Results:The highest incidence of publications occurred in international journals, predominantly cohort studies with level of evidence IV.As for the causes of mortality, cardiovascular disease prevailed.The factors associated with mortality were related to cardiovascular function, infectious, metabolic, physiological, nutritional and structural processes. Conclusions: Improvements in the evaluation and follow-up of renal transplant recipients are recommended.
\end{abstract}

Descriptors: Kidney transplantation; Mortality; Risk factors; Postoperative complications; Review.

'Enfermeiro. Mestre em Enfermagem, Doutorando em Saúde e Desenvolvimento na Região Centro-Oeste pela Universidade Federal de Mato Grosso do Sul/ UFMS - Campo Grande (MS), Brasil. E-mail:will_tlcity@hotmail.com

${ }^{2}$ Enfermeiro. Doutor em Enfermagem, Professor Associado do Curso de Enfermagem da Universidade Federal de Mato Grosso do Sul/UFMS - Três Lagoas (MS), Brasil.E-mail: a.amr@ig.com.br

${ }^{3}$ Enfermeiro. Doutor em Ciências da Saúde. Professor Adjunto do Curso de Enfermagem da Universidade Federal de Mato Grosso do Sul/UFMS - Três Lagoas (MS), Brasil. E-mail: marcelosaude@hotmail.com

${ }^{4}$ Enfermeira. Doutora em Enfermagem. Professor Associado do Curso de Enfermagem da Universidade Federal de Goiás/UFG - Goiânia (GO), Brasil. E-mail: analuciaqueiroz@uol.com.br

${ }^{5}$ Enfermeira. Mestre em Enfermagem. Doutoranda em Saúde e Desenvolvimento na Região Centro-Oeste pela Universidade Federal de Mato Grosso do Sul/ UFMS - Campo Grande (MS), Brasil. E-mail: adaiele@hotmail.com

${ }^{6}$ Enfermeira. Mestre em Enfermagem. Doutoranda em Enfermagem pela Universidade Federal de Goiás/UFG - Goiânia (GO), Brasil. E-mail:cc-teixeira@hotmail.com Artigo baseado no Trabalho de Conclusão de Curso do curso de Especialização em Captação, Doação e Transplante de Órgãos e Tecidos pela Faculdade de Ciências da Saúde Albert Einstein, São Paulo (SP), 2016.

Não há conflitos de interesse

Trabalho realizado com apoio do Programa de Desenvolvimento Institucional do Sistema Único de Saúde (PROADI-SUS). 


\section{INTRODUÇÃO}

O transplante renal é considerado a modalidade de tratamento mais indicado e necessário para pacientes com doença renal crônica, em estágio terminal, e o rim é o órgão mais frequentemente transplantado( ${ }^{(1)}$. Nos países desenvolvidos considera-se que os custos acumulados do transplante renal são inferiores aos dos tratamentos de diálise.Além de proporcionar melhor qualidade de vida e maior sobrevida aos pacientes, comparado ao tratamento dialítico, o transplante também possibilita ter melhor custo-efetividade ${ }^{(2-3)}$.

O paciente transplantado está constantemente, exposto aos riscos de rejeição e por isso dependente do uso diário de medicamentos imunossupressores. Desta forma, é necessário que ele tenha informações suficientes para conviver com a chance de rejeição e com o novo modo de vida, sem as sessões de hemodiálise ${ }^{(4)}$.

Com o aprimoramento progressivo da imunofarmacologia e a qualidade na assistência aos pacientes, observou-se, ao decorrer do tempo menor risco de rejeição e consequentemente, diminuição das taxas de mortalidade após o transplante renal.

Entretanto, a predominância de fatores de risco cardiovasculares como: hipertensão arterial sistêmica (HAS), diabetes mellitus (DM), dislipidemias antes do transplante renal são altas ${ }^{(6)}$ conferindo um risco de morte por doença cardiovascular de 3,5\% a $5 \%$ todos os $\operatorname{anos}^{(7)}$. Assim sendo, as doenças cardiovasculares (DCV) se mantém, como a principal causa dos óbitos após o transplante renal ${ }^{(5)}$.

As infecções são outro fator de risco importante para a mortalidade em transplantados renais, visto que os pacientes possuem alta suscetibilidade a infecções bacterianas, fúngicas e virais decorrentes do uso de imunossupressor ${ }^{(8)}$.

O estudo dos fatores de risco envolvidos na doença e tratamento do doente renal, fornece informações sobre o impacto do processo assistencial do transplantado renal.

Diante da importância desse contexto, objetivou-se analisas as produções científicas sobre os fatores associados à mortalidade em pacientes adultos submetidos ao transplante renal.

\section{MÉTODOS}

Optou-se pela revisão integrativa da literatura científica, cujo método possibilita analisar e sumarizar as pesquisas existentes. ${ }^{9}$ Com a intenção de minimizar possíveis falhas que poderiam influenciar negativamente a qualidade da revisão. Foram percorridas na elaboração do estudo as seguintes etapas: I) definição do problema e formulação da questão norteadora; 2) critérios para seleção da amostra e busca na literatura pertinente; 3 ) levantamento dos dados relevantes a serem extraídos dos estudos selecionados; 4) leitura na íntegra e análise criteriosa dos estudos incluídos; 5) explanação dos resultados, interpretação dos dados e apresentação da revisão/síntese do conhecimento(9-11).

Para a elaboração da questão de pesquisa da revisão integrativa, utilizou-se a estratégia PICO (acrônimo para patient, intervention, comparison, outcomes). $O$ uso dessa estratégia para formular a questão de pesquisa na condução de métodos de revisão, possibilita a identificação de palavras-chave, as quais auxiliam na localização de estudos primários relevantes nas bases de dados ${ }^{(12)}$. Assim, a questão de pesquisa delimitada foi: "Quais causas e fatores estão relacionados à mortalidade no póstransplante renal de pacientes adultos?".

O primeiro elemento da estratégia $(\mathrm{P})$ consiste no paciente transplantado de rim; o segundo (I), os estudos relativos as causas de mortalidade e fatores relacionados; e o quarto elemento $(\mathrm{O})$ causas de mortalidade e fatores relacionados. Ressalta-se que, dependendo do método de revisão, não se emprega todos os elementos da estratégia PICO. Nesta revisão integrativa, o terceiro elemento, ou seja, a comparação, não foi utilizada.

A busca dos estudos ocorreu em julho de 2016, nas seguintes bibliotecas eletrônicas: Biomedical Literature Ciattions and Abstracts Norte-Americana (PubMed), Scientific Electronic Library Online (SciELO) e Cochrane Library e na base de dados Literatura Latino-Americana e do Caribe em Ciências da Saúde (LILACS). Os descritores controlados selecionados foram os seguintes Descritores em Ciências da Saúde (DeCS): "transplante de rim"; "mortalidade"; "fatores de risco", com as respectivas traduções em inglês, padronizadas no MeSH (Medical Subject Heading): "Kidney transplantation"; "mortality"; "risk factors".

Os termos foram combinados de diferentes formas para garantir busca ampla, cujos cruzamentos em todas as bases de dados foram realizados concomitantemente com os operadores booleanos AND e OR os três descritores e palavras na base de dados LILACS ("Transplante de rim") OR "Transplante de rim [Palavras] AND ("Mortalidade") OR “Mortalidade" [Palavras] AND ("Fatores de risco") OR "Fatores de risco" [Palavras]; cruzamentos concomitantemente com o operador booleano AND os MeSH Terms no PubMed ("kidney transplantation"[MeSH Terms] AND "mortality"[MeSH Terms]) AND "risk factors"[MeSH Terms]), cruzamentos concomitantemente com o operador booleano AND todos os índices na biblioteca eletrônica SciELO (Transplante de rim [Todos os índices] AND Mortalidade [Todos os índices] AND Fatores de Risco [Todos os índices]), cruzamentos concomitantemente com o operador booleano AND entre os keywords "kidney transplantation" in 
Keywords and "mortality" in Keywords and "risk factor" in Keywords in Trials' na biblioteca Cochrane

Os critérios de inclusão dos estudos selecionados para esta revisão integrativa foram: artigos completos disponíveis na íntegra nos idiomas português, inglês ou espanhol; estudos sobre pacientes adultos submetidos ao transplante renal; fatores de risco para mortalidade após o transplante; complicações infecciosas que ocorreram até um ano após o transplante renal, e período de publicação de janeiro de 2006 a julho de 2016. Optou-se por considerar a literatura deste período, por se tratar de um tema relevante que só recentemente vem sendo investigado, mas que está gradativamente despertando a atenção dos pesquisadores.

$\mathrm{Na}$ busca inicial, 777 artigos foram encontrados, $07 \mathrm{na}$ base LILACS, 745 no PubMed, 03 no SciELO e 22 na Biblioteca Cochrane. Após a leitura dos títulos e resumos, foram excluídas as publicações duplicadas e feita leitura na íntegra dos estudos selecionados e em conformidade com os critérios de inclusão. Após esta etapa, a amostra compôs-se de 21 estudos, os quais foram apresentadas no fluxograma (Figura I), como recomendado pelo grupo PRISMA ${ }^{(13)}$.

Aos estudos incluídos aplicou-se um instrumento elaborado pelos autores objetivando a extração das seguintes informações: título, autor, ano, objetivos, características metodológicas, resultados, discussão e conclusão. A extração dos dados ocorreu de maneira descritiva conforme apresentado nas pesquisas, ou seja, sem manipulação pelos revisores.

Para avaliar a qualidade dos artigos da amostra, foi utilizada uma classificação de acordo com o nível de evidência ${ }^{(14)}$ conforme descrição:I - revisões sistemáticas ou metanálise de relevantes ensaios clínicos; II - evidências de pelo menos um ensaio clínico randomizado controlado bem delineado; III - ensaios clínicos bem delineados sem randomização; IV - estudos de coorte e de casocontrole bem delineados; $\mathrm{V}$ - revisão sistemática de estu-

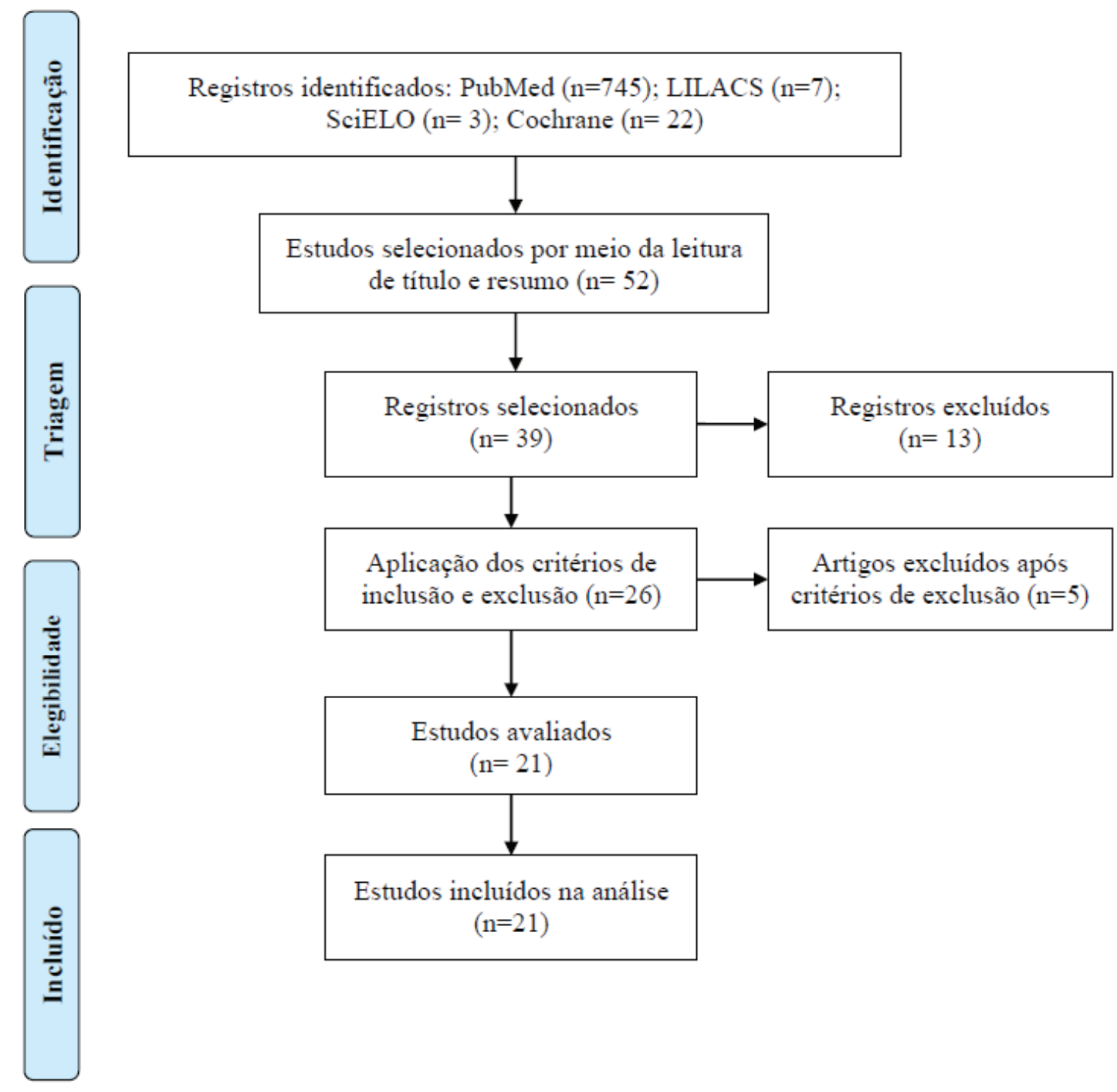

FIGURA 1 - Descrição do processo de seleção dos estudos incluídos na revisão integrativa. São Paulo, SP, Brasil, 2016. 
dos descritivos e qualitativos; $\mathrm{VI}$ - evidências derivadas de um único estudo descritivo ou qualitativo; VII - opinião de autoridades ou comitês de especialistas incluindo interpretações de informações não baseadas em pesquisas.

Os dados extraídos dos artigos estão apresentados de forma descritiva, em dois quadros sinópticos, contendo informações referentes aos títulos do artigo, tipo de estudo, nível de evidência, base de dados, periódico, ano de publicação e tabela descritiva com os fatores associados à mortalidade dos pacientes no pós-transplante renal.

\section{RESULTADOS}

As pesquisas nas bases de dados originaram vinte e um estudos, sendo seis (28,58\%) artigos em 2014 , seguido de quatro (19,05\%) em 2015, não sendo identificado estudos nos anos de 2006 e 2016. Dos vinte e um artigos compilados, dezoito $(85,8 \%)$ são estudos de coorte e destes dez $(47,7 \%)$ são estudos de coorte retrospectivo, e oito $(38,0 \%)$ estudos de coorte prospectivos, com predomínio do nível de evidência IV em $85,8 \%$ dos estudos analisados. A maior incidência de publicações deu-se em periódicos internacionais, com 19 (86,3\%) publicações e duas $(9,5 \%)$ em periódicos nacionais. A avaliação dos periódicos apresentou o total de pacientes receptores de transplante renal, causa de mortalidade e os possíveis fatores relacionados a mortalidade conforme descrito no quadro $I$.

QUADRO 1 - Descrição dos estudos incluídos na revisão integrativa, segundo título, tipo de estudo, nível de evidencia, fonte de dados, periódico, causas e fatores relacionados à mortalidade em receptores de transplante renal e ano de publicação. São Paulo, SP, Brasil, 2016.

\begin{tabular}{|c|c|c|c|c|c|c|c|c|}
\hline $\mathbf{N}$ & Título dos artigos & Tipo de estudo & $\begin{array}{l}\text { Nível de } \\
\text { evidência }\end{array}$ & $\begin{array}{l}\text { Fonte de } \\
\text { dados }\end{array}$ & Periódico & $\begin{array}{l}\text { Causa da } \\
\text { mortalidade }\end{array}$ & $\begin{array}{l}\text { Fatores } \\
\text { Associados à } \\
\text { mortalidade }\end{array}$ & Ano \\
\hline 1 & $\begin{array}{l}\text { Frailty and mortality } \\
\text { in kidney transplant } \\
\text { recipients }\end{array}$ & $\begin{array}{l}\text { Prospectivo } \\
\text { Longitudinal }\end{array}$ & VI & PubMed & $\begin{array}{l}\text { American Journal } \\
\text { of Transplantation }\end{array}$ & - Não descrito & - Fragilidade & 2015 \\
\hline 2 & $\begin{array}{l}\text { Causal path analyses } \\
\text { of the association of } \\
\text { protein intake with risk } \\
\text { of mortality and graft } \\
\text { failure in renal transplant } \\
\text { recipients }\end{array}$ & Coorte Prospectivo & IV & PubMed & $\begin{array}{l}\text { Clinical } \\
\text { Transplantation }\end{array}$ & $\begin{array}{l}\text { - Cardiovascular } \\
\text { - Infecção } \\
\text { - Neoplasia } \\
\text { - Desconhecido } \\
\text { - Outros }\end{array}$ & $\begin{array}{l}\text { - Baixa } \\
\text { ingestão de } \\
\text { proteínas }\end{array}$ & 2015 \\
\hline 3 & $\begin{array}{l}\text { Initial kidney graft } \\
\text { resistance index and the } \\
\text { long-term cardiovascular } \\
\text { mortality in transplanted } \\
\text { patients: a paired grafts } \\
\text { analysis }\end{array}$ & Coorte Prospectivo & IV & PubMed & $\begin{array}{l}\text { Nephrology } \\
\text { Dialysis } \\
\text { Transplantation }\end{array}$ & - Cardiovascular & $\begin{array}{l}\text { - Aumento } \\
\text { do índice de } \\
\text { resistência intra- } \\
\text { renal }\end{array}$ & 2015 \\
\hline 4 & $\begin{array}{l}\text { Evolução a longo prazo } \\
\text { no transplante renal de } \\
\text { idosos }\end{array}$ & $\begin{array}{l}\text { Coorte } \\
\text { Retrospectivo }\end{array}$ & IV & SciELO & $\begin{array}{l}\text { Jornal Brasileiro } \\
\text { de Nefrologia }\end{array}$ & $\begin{array}{l}\text { - Infecção } \\
\text { - Cardiovascular } \\
\text { - Neoplasia } \\
\text { - Outros } \\
\text { - Desconhecido }\end{array}$ & $\begin{array}{l}\text { - Diabetes } \\
\text { Mellitus em } \\
\text { pacientes } \\
\text { maiores de } 60 \\
\text { anos }\end{array}$ & 2015 \\
\hline 5 & $\begin{array}{l}\text { The impact of early } \\
\text { cytomegalovirus } \\
\text { infection after kidney } \\
\text { transplantation on long- } \\
\text { term graft and patient } \\
\text { survival }\end{array}$ & Coorte Prospectivo & IV & PubMed & $\begin{array}{l}\text { Clinical } \\
\text { Transplantation }\end{array}$ & $\begin{array}{l}\text { - Cardiovascular } \\
\text { - Neoplasia } \\
\text { - Infecção } \\
\text { - Outras causas }\end{array}$ & $\begin{array}{l}\text { - Infecção por } \\
\text { Citomegalovírus } \\
(\mathrm{CMV})\end{array}$ & 2014 \\
\hline 6 & $\begin{array}{l}\text { Association of pre- } \\
\text { transplant blood pressure } \\
\text { with posttransplant } \\
\text { outcomes }\end{array}$ & Coorte Prospectivo & IV & PubMed & $\begin{array}{l}\text { Clinical } \\
\text { Transplantation }\end{array}$ & - Cardiovascular & $\begin{array}{l}\text { - Aumento da } \\
\text { pressão arterial } \\
\text { pré-transplante }\end{array}$ & 2014 \\
\hline
\end{tabular}




\begin{tabular}{|c|c|c|c|c|c|c|c|c|}
\hline 7 & $\begin{array}{l}\text { Clopidogrel use as } \\
\text { a risk factor for poor } \\
\text { outcomes after kidney } \\
\text { transplantation }\end{array}$ & $\begin{array}{l}\text { Coorte } \\
\text { Retrospectivo }\end{array}$ & IV & PubMed & $\begin{array}{l}\text { The American } \\
\text { Journal of Surgery }\end{array}$ & - Não descrito & $\begin{array}{l}\text { - Uso de } \\
\text { clopidogrel até } \\
90 \text { ou mais } \\
\text { de } 90 \text { dias } \\
\text { combinado } \\
\text { com doença da } \\
\text { artéria coronária, } \\
\text { doença } \\
\text { cerebrovascular } \\
\text { e/ou doença } \\
\text { vascular } \\
\text { periférica. }\end{array}$ & 2014 \\
\hline 8 & $\begin{array}{l}\text { Mortality predictors } \\
\text { in renal transplant } \\
\text { recipients with severe } \\
\text { sepsis and septic shock }\end{array}$ & $\begin{array}{l}\text { Retrospectivo } \\
\text { Observacional }\end{array}$ & $\mathrm{V}$ & PubMed & $\begin{array}{l}\text { Public Library of } \\
\text { Science one }\end{array}$ & - Choque séptico & $\begin{array}{l}\text { - Sexo } \\
\text { masculino } \\
\text { - Admissão das } \\
\text { enfermarias } \\
\text { - Score SOFA } \\
\text { piores no } \\
\text { primeiro dia } \\
\text { - Disfunção } \\
\text { hematológica } \\
\text { - Ventilação } \\
\text { Mecânica } \\
\text { - Disfunção } \\
\text { tardia do enxerto }\end{array}$ & 2014 \\
\hline 9 & $\begin{array}{l}\text { Recipient-related risk } \\
\text { factors for graft failure } \\
\text { and death in elderly } \\
\text { kidney transplant } \\
\text { recipients }\end{array}$ & $\begin{array}{l}\text { Coorte } \\
\text { Retrospectivo }\end{array}$ & IV & PubMed & $\begin{array}{l}\text { Public Library of } \\
\text { Science one }\end{array}$ & $\begin{array}{l}\text { - Infecção } \\
\text { - DAC* } \\
\text { - } \mathrm{AVC}^{\dagger} \\
\text { - Neoplasia } \\
\text { - Doença } \\
\text { Hepática } \\
\text { - Hemorragia } \\
\text { - Desconhecido }\end{array}$ & $\begin{array}{l}\text { - Dose baixa } \\
\text { na profilaxia } \\
\text { com ganciclovir } \\
\text { - Longo tempo } \\
\text { de diálise }\end{array}$ & 2014 \\
\hline 10 & $\begin{array}{l}\text { Symmetric } \\
\text { dimethylarginine as } \\
\text { predictor of graft loss } \\
\text { and all-cause mortality } \\
\text { in renal transplant } \\
\text { recipients }\end{array}$ & $\begin{array}{l}\text { Randomizado, } \\
\text { duplo-cego, } \\
\text { placebo-controlado }\end{array}$ & ॥ & $\begin{array}{l}\text { Cochrane } \\
\text { Database }\end{array}$ & Transplantation & $\begin{array}{l}\text { - Cardiovascular } \\
\text { - Infecção } \\
\text { - Neoplasia }\end{array}$ & $\begin{array}{l}\text { - Aumento } \\
\text { dimetilarginina } \\
\text { simétrica }\end{array}$ & 2014 \\
\hline 11 & $\begin{array}{l}\text { Predictors of graft } \\
\text { failure and death in } \\
\text { elderly kidney transplant } \\
\text { recipients }\end{array}$ & Coorte Prospectivo & IV & PubMed & $\begin{array}{l}\text { Clinical and } \\
\text { Translational } \\
\text { Research }\end{array}$ & - Não descrito & $\begin{array}{l}\text { - Insuficiência } \\
\text { Cardíaca } \\
\text { Congestiva } \\
\text { e Doença } \\
\text { da Artéria } \\
\text { Coronária em } \geq \\
65 \text { anos }\end{array}$ & 2013 \\
\hline 12 & $\begin{array}{l}\text { Rate of renal graft } \\
\text { function decline after one } \\
\text { year is a strong predictor } \\
\text { of all-cause mortality }\end{array}$ & $\begin{array}{l}\text { Retrospectivo } \\
\text { Longitudinal }\end{array}$ & IV & PubMed & $\begin{array}{l}\text { American Journal } \\
\text { of Transplantation }\end{array}$ & $\begin{array}{l}\text { - Cardiovascular } \\
\text { - Neoplasia } \\
\text { - Infecção } \\
\text { - Hepatopatia } \\
\text { - Má nutrição } \\
\text { - Diabete } \\
\text { Mellitus } \\
\text { - Respiratório } \\
\text { - Desconhecido }\end{array}$ & $\begin{array}{l}\text { - Diminuição } \\
\text { da taxa de } \\
\text { filtração } \\
\text { glomerular }\end{array}$ & 2013 \\
\hline 13 & $\begin{array}{l}\text { Left atrial diameter and } \\
\text { survival among renal } \\
\text { allograft recipients }\end{array}$ & $\begin{array}{l}\text { Coorte } \\
\text { Retrospectivo }\end{array}$ & IV & PubMed & $\begin{array}{l}\text { Clinical Journal } \\
\text { of the American } \\
\text { Society of } \\
\text { Nephrology }\end{array}$ & $\begin{array}{l}\text { - Cardiovascular } \\
\text { - Infecção } \\
\text { - Neoplasia } \\
\text { - Outros }\end{array}$ & $\begin{array}{l}\text { - Diâmetro do } \\
\text { átrio esquerdo } \\
\text { alargado-Morte } \\
\text { cardíaca }\end{array}$ & 2013 \\
\hline
\end{tabular}




\begin{tabular}{|c|c|c|c|c|c|c|c|c|}
\hline 14 & $\begin{array}{l}\text { Endogenous plasma } \\
\text { erythropoietin, } \\
\text { cardiovascular mortality } \\
\text { and all-cause mortality } \\
\text { in renal transplant } \\
\text { recipients }\end{array}$ & Coorte Prospectivo & IV & PubMed & $\begin{array}{l}\text { American Journal } \\
\text { of Transplantation }\end{array}$ & $\begin{array}{l}\text { - Cardiovascular } \\
\text { - Outros }\end{array}$ & $\begin{array}{l}\text { - Aumento } \\
\text { das taxas de } \\
\text { eritropoetina }\end{array}$ & 2012 \\
\hline 15 & $\begin{array}{l}\text { Análise da sobrevida } \\
\text { e fatores associados } \\
\text { à mortalidade em } \\
\text { receptores de } \\
\text { transplante renal em } \\
\text { Hospital Universitário no } \\
\text { Maranhão }\end{array}$ & $\begin{array}{l}\text { Retrospectivo } \\
\text { Observacional }\end{array}$ & IV & PubMed & $\begin{array}{l}\text { Jornal Brasileiro } \\
\text { de Nefrologia }\end{array}$ & $\begin{array}{l}\text { - Choque séptico } \\
\text { - Cardiovascular } \\
\text { - Choque } \\
\text { hipovolêmico } \\
\text { - Toxoplasmose } \\
\text { cerebral } \\
\text { - Varicela } \\
\text { - Câncer de } \\
\text { pulmão }\end{array}$ & $\begin{array}{l}\text { - Idade > que } \\
40 \text { anos } \\
\text { - Intercorrência } \\
\text { Cirúrgica }\end{array}$ & 2012 \\
\hline 16 & $\begin{array}{l}\text { Serum phosphate and } \\
\text { calcium concentrations } \\
\text { are associated with } \\
\text { reduced patient survival } \\
\text { following kidney } \\
\text { transplantation }\end{array}$ & Coorte Prospectivo & IV & PubMed & $\begin{array}{l}\text { Clinical } \\
\text { Transplantation }\end{array}$ & - Outros & $\begin{array}{l}\text { - Níveis } \\
\text { elevados de } \\
\text { fosfato e de } \\
\text { cálcio no soro. }\end{array}$ & 2011 \\
\hline 17 & $\begin{array}{l}\text { The association of early } \\
\text { post-transplant glucose } \\
\text { levels with long-term } \\
\text { mortality }\end{array}$ & Coorte Prospectivo & IV & PubMed & Diabetologia & $\begin{array}{l}\text { - Cardiovascular } \\
\text { - Neoplasia } \\
\text { - Infecção } \\
\text { - Outros } \\
\text { - Desconhecido }\end{array}$ & - Hiperglicemia & 2011 \\
\hline 18 & $\begin{array}{l}\text { Risk factors associated } \\
\text { with graft loss and } \\
\text { patient survival after } \\
\text { kidney transplantation }\end{array}$ & $\begin{array}{l}\text { Coorte } \\
\text { Retrospectivo }\end{array}$ & IV & PubMed & $\begin{array}{l}\text { Transplantation } \\
\text { Proceedings }\end{array}$ & - Não descrito & - Não descrito & 2009 \\
\hline 19 & $\begin{array}{l}\text { Associations between } \\
\text { pre-kidney-transplant } \\
\text { risk factors and post- } \\
\text { transplant cardiovascular } \\
\text { events and death }\end{array}$ & $\begin{array}{l}\text { Coorte } \\
\text { Retrospectivo }\end{array}$ & IV & PubMed & $\begin{array}{l}\text { Transplant } \\
\text { International }\end{array}$ & - Não descrito & $\begin{array}{l}\text { - Idade } \\
\text { Avançada } \\
\text { - Histórico } \\
\text { de evento } \\
\text { cardiovascular } \\
\text { - Doença } \\
\text { cerebrovascular } \\
\text { - Nefropatia } \\
\text { Diabética }\end{array}$ & 2008 \\
\hline 20 & $\begin{array}{l}\text { The association between } \\
\text { recipient alcohol } \\
\text { dependency and long- } \\
\text { term graft and recipient } \\
\text { survival }\end{array}$ & $\begin{array}{l}\text { Coorte } \\
\text { Retrospectivo }\end{array}$ & IV & PubMed & $\begin{array}{l}\text { Nephrology, } \\
\text { Dialysis, } \\
\text { Transplantation }\end{array}$ & - Não descrito & $\begin{array}{l}\text { - Dependência } \\
\text { de álcool na } \\
\text { doença renal } \\
\text { terminal }\end{array}$ & 2007 \\
\hline 21 & $\begin{array}{l}\text { Decreased serum } \\
\text { retinol is associated } \\
\text { with increased mortality } \\
\text { in renal transplant } \\
\text { recipients }\end{array}$ & $\begin{array}{l}\text { Coorte } \\
\text { Retrospectivo }\end{array}$ & IV & PubMed & Clinical Chemistry & $\begin{array}{l}\text { - Cardiovascular } \\
\text { - Não } \\
\text { cardiovascular } \\
\text { - Desconhecido }\end{array}$ & $\begin{array}{l}\text { - Baixos níveis } \\
\text { de Retinol } \\
\text { (Vitamina A) }\end{array}$ & 2007 \\
\hline
\end{tabular}

*DAC - Doença da artéria coronária; ${ }^{\dagger} \mathrm{AVC}$ - Acidente Vascular Cerebral

Quanto as causas da mortalidade prevaleceram a cardiovascular em treze $(61,91 \%)$ estudos, seguido da infecção em nove $(42,9 \%)$ artigos analisados. Conforme aparecem nas investigações, o número de óbitos por causas cardiovasculares e não cardiovasculares são descritas na tabela I, baseado em todos os óbitos descritos nos estudos analisados.

\section{DISCUSSÃO}

Quanto ao local de origem dos artigos selecionados, foram produzidos predominantemente na América do Norte e Europa.

Quanto ao tipo de estudo utilizado, a maioria dos artigos foi composta por estudos de coorte, no qual existe a 
TABELA 1 - Máximo, mínimo, média desvio padrão (Mediana) das causas de mortalidade de pacientes receptores de transplante renal conforme descrito no quadro 3. São Paulo, SP, Brasil, 2016. $(n=21)$

\begin{tabular}{l|c|c|c|c}
\hline \multicolumn{1}{c|}{ Variáveis } & Max & Min & Média \pm DP & Mediana \\
\hline Causas da mortalidade & & & & \\
\hline Cardiovascular & 194,00 & 0,00 & $36,67 \pm 49,99$ & 14,00 \\
\hline Infecção & 76,00 & 0,00 & $18,00 \pm 25,96$ & 0,00 \\
\hline Neoplasia & 65,00 & 0,00 & $10,71 \pm 18,50$ & 0,00 \\
\hline Outros & 22,00 & 0,00 & $2,66 \pm 5,79$ & 0,00 \\
\hline Desconhecido & 57,00 & 0,00 & $12,66 \pm 17,83$ & 4,00 \\
\hline
\end{tabular}

dificuldade para o controle de vieses ${ }^{(15)}$, salienta-se que, os estudos de coorte são utilizados de maneira clássica para avaliar resultados de exposições a riscos ${ }^{(16)}$. Deste modo, diante à avaliação dos fatores de risco para a mortalidade no pós-transplante renal, este seria um dos delineamentos mais recomendados para este tipo de investigação.

Os fatores de riscos de mortalidade de receptores de transplante renal foram distribuídos em categorias conforme a frequência, quais sejam: Fragilidade; Nutrição; Diabetes; Infecção; Cardiovascular e Infecção.

A fragilidade foi avaliada prospectivamente em 537 receptores de transplante renal, mostrando que, após cinco anos do transplante, os sobreviventes foram $91,5 \%$, $86,0 \%$ e $77,5 \%$ para os receptores de transplante renal não frágeis, intermediários e frágeis, respectivamente e concluiu que independentemente da idade, a fragilidade é um forte e independente fator de risco para a mortalidade pós transplante renal. Estudos que avaliaram outras situações como, pacientes de todas as idades submetidos à hemodiálise e idosos submetidos a intervenções coronarianas percutâneas mostraram que a fragilidade foi associada a um risco aumentado de mortalidade naqueles pacientes que eram frágeis ${ }^{(17-18)}$.

A parte nutricional do receptor de transplante renal tem mostrado ser um importante fator na sobrevida destes pacientes. Um estudo verificou que uma ingestão relativamente elevada de proteínas está associada a um risco reduzido de mortalidade e de falência do enxerto em pacientes transplantados ${ }^{(19)}$. Uma coorte de 940 receptores de transplante renal mostrou que a ingestão de proteína está inversamente associada com a mortalidade e a falência do enxerto ${ }^{(20)}$. Outro estudo que avaliou os níveis séricos de vitamina $A$, identificou que a baixa concentração de retinol (Vitamina A) está significativamente associada à mortalidade por todas as causas em pacientes com transplante renal, uma vez que estes pacientes estão em uso de imunossupressores e estão muito mais suscetíveis as infecções ${ }^{(21)}$. Um estudo mostrou que as concentrações inadequadas de vitamina $A$ intensificam a gravidade de processos infecciosos ${ }^{(22)}$ ocasionando maiores chances de mortalidade.
Várias são as comorbidades relacionadas à perda precoce do enxerto e mortalidade no pós-transplante renal, dentre elas o DM. O impacto da hiperglicemia na sobrevivência do doente após o transplante renal é desconhecido. Alguns estudos indicam uma associação entre diabetes mellitus pós-transplante e mortalidade ${ }^{(23-25)}$, enquanto outros não ${ }^{(26-27)}$. Um estudo mostrou que a Síndrome metabólica e o diabetes mellitus pós-transplante foram fortemente associadas, mas não permitiu estabelecer qualquer associação possível entre diabetes mellitus póstransplante e sobrevivência renal e/ou paciente ${ }^{28}$ enquanto que outros estudos apontam a redução da sobrevida do enxerto e/ou do paciente, na vigência de diabetes mellitus pós-transplante ${ }^{(29-30)}$, o que mostra a necessidade de mais estudos a fim de identificar os efeitos diretos do diabetes sobre os pacientes receptores de transplante renal.

A prevalência de fatores de risco cardiovascular em candidatos a transplante renal é alta; um estudo realizado com 50 pacientes renais crônicos apontou que $78 \%$ dos participantes eram hipertensos, sendo a hipertensão umas das principais causas de insuficiência renal crônica ${ }^{(31)}$. Uma melhor compreensão da relação entre esses fatores de risco e morbidade e mortalidade cardiovascular é necessária para melhorar o resultado do transplante. Nefropatia diabética e doença cardiovascular foram importantes preditores de mortalidade por todas as causas sendo os mais importantes para eventos cardiovasculares e mortalidade por todas as causas após transplante renal ${ }^{(32)}$.

Outro fator importante associado à mortalidade é a relação da pressão arterial onde baixa pressão sistólica pós-diálise e a diastólica baixa pré-diálise estão associadas a menor risco de morte pós-transplante, enquanto a pressão diastólica pós-diálise muito elevada está associada a maior mortalidade em receptores de transplante renal. As variações da pressão arterial de pacientes em diálise antes do transplante renal podem ter influência no desfecho pós-transplante, o que justifica estudos adicionais $^{(33)}$. Vários fatores de risco pré-transplante foram identificados nos últimos anos, mas a associação entre a pressão arterial pré-transplante e a mortalidade póstransplante não haviam sido previamente avaliada.Apon- 
tam-se que devido à falta de dados, a maioria das diretrizes sobre a avaliação de candidatos a transplante renal não incluiu recomendações sobre a pressão arterial ideal de pacientes na lista de espera ${ }^{(34-37)}$.

A recente recomendação da Sociedade Americana de Transplante mencionou que a HAS (PA $\geq 140 / 90 \mathrm{~mm}$ $\mathrm{Hg}$ ou tomando medicamentos anti-hipertensivos) é um fator de risco de cardiopatia isquêmica e outras doenças ateroscleróticas, mas não forneceu uma faixa ideal de pressão arterial durante o período de espera ${ }^{(35)}$.A Diretriz Europeia de Melhores Práticas e a orientação de avaliação do Reino Unido não apontaram dados atualizados referentes a pressão arterial em pacientes em diálise na lista de espera ${ }^{(36-37)}$.

Recentemente um estudo analisou o efeito da resistência intra-renal e revelou maior mortalidade cardiovascular e todas as causas entre os pacientes com valores iniciais de resistência intra-renal mais elevados, sendo um forte preditor de morte cardiovascular, independente dos episódios cardiovasculares anteriores. $O$ achado mais importante é o fato de que entre os pacientes transplantados com rins adquiridos a partir do mesmo doador, aqueles com valores de resistência intra-renal mais altos medidos no enxerto renal durante os primeiros dias após o transplante apresentam um risco significativamente maior de morte cardiovascular durante o longo tempo observação ${ }^{(38)}$.

Estudos tem apontado a relação de infecção pelo citomegalovírus (CMV) com a mortalidade em pacientes receptores de transplante renal. Um estudo de coorte observacional prospectivo relatou efeitos do CMV sobre a sobrevivência do enxerto e do paciente em 47I receptores de transplante renal entre 1994 e 1997. Nenhum dos pacientes recebeu profilaxia para o citomegalovírus ou tratamento preventivo. Durante uma mediana de I3,7 (7,I-I4,9) anos, o número de perdas de enxerto censuradas por morte foi de 118 (25\%) e de mortes de pacientes 224 (48\%). Na análise univariada, a infecção por CMV foi significativamente associada à perda de enxerto censurado por morte, sugerindo que a infecção precoce pelo citomegalovírus está associada a um aumento da mortalidade global a longo prazo ${ }^{(39)}$.

Outro estudo que analisou fatores de risco para morte e perda do enxerto receptores de transplante renal descobriu que pacientes mesmo em uso de ganciclovir para profilaxia de infecções causados pelo citomegalovírus em doses baixas foi fator de risco para a mortalidade em receptores com idade $>60$ anos. Este fator representa potencial alvo para intervenções destinadas a melhorar a sobrevivência do enxerto e do doente em receptores idosos. Segundo o mesmo estudo a profilaxia com ganciclovir em altas doses deve ser amplamente utilizada em receptores idosos para diminuir a incidência de infecção por citomegalovírus. No entanto, estudos prospectivos, randomizados e multicêntricos são necessários para confirmar esses achados ${ }^{(40)}$.

Limitações do estudo estão relacionadas ao período de coleta de dados e ao número reduzido de bases de dados, dificultando a generalização dos resultados. Sugere-se a realização de outros estudos desta natureza, no sentido de gerar novos conhecimentos sobre a incidência de complicações e mortalidade do transplante renal, bem como das estratégias de prevenção.

\section{CONCLUSÃO}

Este estudo permitiu concluir que diversos são os fatores associados à mortalidade em receptores de transplante renal, sendo estes, fatores relacionados à função cardiovascular, processos infecciosos, metabólicos, fisiológicos, nutricionais e estruturais. As investigações relacionadas ao transplante renal permitem nortear novos estudos para propor melhorias no processo de avaliação e acompanhamento dos pacientes receptores de transplante renal. Por este motivo o presente estudo pode ter implicações que possam instigar a novas pesquisas, a fim de determinar as melhores recomendações para garantir a sobrevida do enxerto e do receptor, contribuindo para melhor qualidade de vida do paciente. 


\section{REFERÊNCIAS}

I. British Transplant Society/Renal Association. United kingdom guidelines for living donor kidney transplantation. 3a.ed. Londres: BTS/RA; $201 \mathrm{I}$.

2. Farney AC, Rogers J, Orlando G, al-Geizawi S, Buckley M, Farooq $U$, et al. Evolving experience using kidneys from deceased donors with terminal acute kidney injury. J Am Col Surg. 2013;216(4):645-55.

3. Wolfe RA, Ashby VB, Milford EL, Ojo AO, Ettenger RE, Agodoa LY, et al. Comparison of mortality in all patients on dialysis, patients on dialysis awaiting transplantation, and recipients of a first cadaveric transplant. $\mathrm{N}$ Engl J Med. I 999;34 I (23): I 725-30.

4. Lira ALBC, Lopes MVO. Pacientes transplantados renais: análise de associação dos diagnósticos de enfermagem. Rev. Gaúcha Enferm. 2010;3।(I):108-14.

5. Salermo MP, Zichichi E, Rossi E, Favi E, Gargiulo A, Spagnoletti $\mathrm{G}$, et al. Evolution of causes of mortality in renal transplantation in the last 10 years. Transplant Proc. 2010;42: 1077-9.

6. Fellström B, Jardine AG, Soveri I, Cole E, Neumayer $\mathrm{HH}$, Maes $B$, et al. Renal dysfunction is a strong and independent risk factor for mortality and cardiovascular complications in renal transplantation. Am J Transplant. 2005;5: 1986-91.

7. Ojo AO. Cardiovascular complications after renal transplantation and their prevention. Transplantation. 2006;82:603-I I.

8. Fishman JA. Infection in solid-organ transplant recipients. $N$ Engl J Med. 2007; 357:260 I- I4.

9. Mendes KDS, Silveira RCCP, Galvão CM. Revisão integrativa: método de pesquisa para a incorporação de evidências na saúde e na enfermagem. Texto e contexto enferm. 2008; I 7(4):758-64.

10. Souza MT, Silva MD, Carvalho R. Revisão integrativa: o que é e como fazer. Einstein. 2010;8:102-6.

I I. Teixeira E, Medeiros HP, Nascimento MHM, Silva BAC, Rodrigues. Revisão Integrativa da Literatura passo-a-passo e convergências com outros métodos de revisão. Rev Enferm UFPI. 20I3;2(spe): 3-7.

I2. Fineout-Overholt E, Stillwell SB. Asking compelling, clinical questions. In: Melnyk BM, Fineout-Overholt E. Evidence-based practice in nursing \& healthcare: a guide to best practice. Philadelphia:Wolters Kluwer, Lippincot Williams \& Wilkins; 20I I. p. 25-39.

13. Moher D, Liberati A, Tetzlaff J, Altman DG; PRISMA Group. Preferred reporting items for systematic reviews and meta-analyses: the PRISMA Statement. PLoS Med. 2009;6(6):el 000097.

14. Melnyk BM, Fineout-Overholt E. Making the case for evidence-based practice. In: Melnyk BM, Fineout-Overholt E. Evidence-based practice in nursing \& healthcare: a guide to best practice. Philadelphia: Lippincot Williams \& Wilkins; 20I I, p. 3-24.

15. Carlson MDA, Morrison RS. Study Design, Precision, andValidity in Observational Studies.J Palliat Med. 2009; 12 (I):77-82.
16. Lazcano-Ponce E, Fernández E, Salazar-Martínez E, Hernández-Ávila M. Estudios de cohorte. Metodología, sesgos y aplicación. Salud Pública Méx. 2000;42(3):230-4I.

17. McAdams-Demarco MA, Law A, Salter M, Boyarsky B, Gimenez L, Jaar BG, et al. Frailty as a novel predictor of mortality and hospitalization in hemodialysis patients of all ages. J Am Geriatr Soc. 20 I 3;6I(6):896-90I.

18. Singh M, Rihal CS, Lennon RJ, Spertus JA, Nair KS, Roger $\mathrm{VL}$. Influence of frailty and health status on outcomes in patients with coronary disease undergoing percutaneous revascularization. Circ Cardiovasc Qual Outcomes. 201 I;4(5):496-502.

19. Said MY, Deetman PE, de Vries AP, Zelle DM, Gans RO, Navis $\mathrm{G}$, et al. Causal path analyses of the association of protein intake with risk of mortality and graft failure in renal transplant recipients. Clin Transplant. 20 I5;29(5):447-57.

20. Deetman PE Said MY, Kromhout D, Dullaart RP, Kootstra -Ros JE, Sanders JS, et al. Urinary urea excretion and longterm outcome after renal transplantation. Transplantation. 20I5;99(5): I009-I5.

21. Connolly GM, Cunningham R, Maxwell AP,Young IS. Decreased serum retinol is associated with increased mortality in renal transplant recipients. Clin Chem. 2007;53(I0): I84 I-6.

22. Cribb VL, Northstone K, Hopkins D, Emmett PM. Sources of vitamin $A$ in the diets of pre-school children in the Avon Longitudinal Study of Parents and Children (ALSPAC). Nutrients. 20I 3;5:1609-2I.

23. Orlandi PF, Cristelli MP, Aldworth CAR, Freitas TVS, Felipe $\mathrm{CR}$, Silva Junior HT, et al. Evolução a longo prazo no transplante renal de idosos.J Bras Nefrol. 20 I5;37(2):2I 2-220.

24. Valderhaug TG, Hjelmesæth J, Hartmann A, Røislien J, Bergrem HA, Leivestad T, et al. The association of early posttransplant glucose levels with long-term mortality. Diabetologia. 201 I;54(6): I 34 I-1349.

25. Aalten J, Hoogeveen EK, Roodnat JI, Weimar W, Borm GF, de Fijter JW, et al. Associations between pre-kidney-transplant risk factors and post-transplant cardiovascular events and death. Transpl Int. 2008;2I (10):985-9I.

26. Hjelmesaeth J, Hartmann A, Leivestad T, Holdaas H, Sagedal $S$, Olstad $M$, et al. The impact of early-diagnosed new-onset post-transplantation diabetes mellitus on survival and major cardiac events. Kidney Int. 2006;69(3):588-95.

27. Miles AM, Sumrani N, Horowitz R, Homel P, Maursky V, Markell MS, et al. Diabetes mellitus after renal transplantation: as deleterious as non-transplantassociated diabetes? Transplantation. 1998; 15;65(3):380-4.

28. Teixeira APSF, Fernandes NMS, Mata GF, Chaoubah A, Paula RB, Bastos MG. Prevalence of metabolic syndrome and its associated factors in renal transplant recipients. J Bras $\mathrm{Ne}$ frol. 2012;34(I): 16-2I.

29. Crutchlow MF, Bloom RD. Transplant-Associated Hyperglycemia: A New Look at an Old Problem. Clin J Am Soc Nephrol. 2007;2(2):343-355. 
30. Van Laecke S, Van Biesen W, Verbeke F, De Bacquer D, Peeters P, Vanholder R. Posttransplantation Hypomagnesemia and Its Relation with Immunosuppression as Predictors of New-Onset Diabetes after Transplantation. Am J Transplant. 2009;9(9):2। 40-9.

31. Fidelis Cl, Balbino CM, Souza MMT, Rodrigues LMS, Silvino ZR, Passos JR. Dificuldades enfrentadas pelo paciente renal para a realização do tratamento. Rev Enferm Atual. 2016; 77:16-2I.

32. Aalten J, Hoogeveen EK, Roodnat JI, Weimar W, Borm GF, De Fijter JW, et al.Associations between pre-kidney-transplant risk factors and post-transplant cardiovascular events and death. Transpl Int. 2008;2 I (I0):985-9I.

33. Molnar MZ, Foster CE, Sim JJ, Remport A, Krishnan M, Kovesdy CP. Association of Pre-Transplant Blood Pressure with Post-Transplant Outcomes. Clin Transplant. 20। 4;28(2): I66-I76.

34. Pilmore H. Cardiac assessment for renal transplantation. American journal of transplantation: official journal of the American Society of Transplantation and the American Society of Transplant Surgeons. 2006;6(4):659.
35. Kasiske BL, Cangro CB, Hariharan S, Hricik DE, Kerman $\mathrm{RH}$, Roth $\mathrm{D}$, et al. The evaluation of renal transplantation candidates: clinical practice guidelines. American journal of transplantation: official journal of the American Society of Transplantation and the American Society of Transplant Surgeons. 200 I; I (Suppl 2):3.

36. Dudley, C.; Harden, P.Assessment of the Potential Kidney Transplant Recipient. 5th Edition ed. UK Renal Association. 201 I.

37. European Best Practice Guidelines for Renal Transplantation (part I). Nephrol Dial Transplant. 2000; I5(Suppl 7): I.

38. Kolonko A, Chudek J, Więcek A. Initial kidney graft resistance index and the long-term cardiovascular mortality in transplanted patients: a paired grafts analysis. Nephrol Dial Transplant. 2015;30(7): I 2 18-24.

39. Smedbraten YV, Sagedal S, Leivestad T, Mjøen G, Osnes K, Rollag $\mathrm{H}$, et al. The impact of early cytomegalovirus infection after kidney transplantation on long-term graft and patient survival. Clin Transplant. 20 I4;28(I):I20-6.

40. Lai X, Chen G, Qiu J, Wang C, Chen L. Recipient-related risk factors for graft failure and death in elderly kidney transplant recipients. PLoS One. 20 I4;9(I I):e I I 2938. 